ISSN: 2174-5609

DOI. https://doi.org/10.14198/INTURI2022.23.5

Cita bibliográfica: Solano Sánchez, M.A., Núñez Tabales, J.M.y Caridad Y López del Río, L. (2022). Nuevos tipos de alojamiento: apartamentos turísticos y determinantes de valoración en el precio de la estancia. Investigaciones Turísticas (23), pp. 94-111. https://doi.org/10.14198/INTURI2022.23.5

\title{
Nuevos tipos de alojamiento: apartamentos turísticos y determinantes de valoración en el precio de la estancia
}

New types of accommodation: Tourist apartments and determining factors of the daily rate

Miguel Ángel Solano Sánchez (D), Universidad de Córdoba, España d42sosam@uco.es

Julia M. Núñez Tabales (iD, Universidad de Córdoba, España

es2nutaj@uco.es

Lorena Caridad y López del Río (iD, Universidad de Córdoba, España d92calol@uco.es

\section{RESUMEN}

El reciente cambio de paradigma en el sector turístico debido al uso generalizado de las tecnologías de la comunicación ha traído consigo un incremento en la facilidad para efectuar reservas por parte del consumidor. Asimismo, antes de la irrupción del COVID-19, se ha asistido a un sostenido incremento en el número de turistas acompañado del aumento en la oferta de distintos tipos de alojamiento turístico, entre los que cabe destacar nuevas modalidades alejadas de los convencionales hoteles, tales como los apartamentos turísticos. El objetivo de este estudio es conocer los determinantes de valoración del precio de la estancia diaria de esta nueva modalidad de alojamiento mediante un modelo econométrico utilizando el método de precios hedónicos y empleando a Booking.com como principal fuente de datos. La ciudad seleccionada para el análisis ha sido Sevilla debido al notable auge de este tipo de alojamiento que ha experimentado en años recientes y por ser la urbe de mayor tamaño del sur de España. Variables referidas a la tipología del inmueble, su ubicación, su tamaño, sus amenidades, así como factores relacionados con la estacionalidad y los eventos especiales de la ciudad aparecen como relevantes en el modelo. El grado de ajuste obtenido en el mismo es alto, así como su poder predictivo, pudiendo ser útil para estimar el precio de la estancia por parte de empresarios, clientes, así como las distintas plataformas online en las que se comercializa esta modalidad de alojamiento, una vez conocidas un conjunto de características internas del inmueble, de su entorno y de estacionalidad.

Palabras clave: Alojamientos turísticos; apartamentos; precios hedónicos; valoración estancia; Booking.com. 


\section{ABSTRACT}

The recent paradigm shift in the tourism sector due to the widespread use of communication technologies has facilitated the booking process for consumers. Similarly, before the outbreak of COVID-19, there was a sustained increase in the number of tourists accompanied by an increase in the offer of different types of tourist accommodation, including new types other than conventional hotels, such as tourist apartments. The aim of this research is to reveal the determinants of the price of the daily rate in this new type of accommodation based on an econometric model using the hedonic price method and using Booking.com as the main source of data. The city selected for the analysis is Seville due to the notable increase in this type of accommodation experienced in the city in recent years and because it is the largest city in Southern Spain. Variables referring to the type of property, its location, its size, its amenities, and factors related to seasonality and special events in the city appear as relevant in the model. The degree of adjustment obtained in the model is high, as is its predictive power and it could be useful for estimating the daily rate for hosts, clients and the different online platforms through which this type of accommodation is marketed, after determining a series of internal characteristics of the property, and those relating to its environment and seasonality.

Key words: Tourist accommodation; apartments; hedonic prices; daily rate valuation; Booking.com.

\section{INTRODUCCIÓN}

El auge de Internet en la era de las comunicaciones ha facilitado los sistemas de oferta y reserva de todo tipo de alojamientos turísticos en determinadas plataformas online tales como Booking.com o Airbnb. Ante un incremento del número de turistas a nivel mundial en la era pre-COVID-19 -un 56\% para el periodo 2009-2019, según UNWTO (2019)- se ha producido un consecuente desarrollo de nuevos tipos de alojamiento para acoger a esa demanda. Dentro de estas nuevas modalidades de alojamientos, es especialmente destacable el caso de los denominados apartamentos turísticos (AT), consistentes en grupos de inmuebles independientes (generalmente adosados unos a otros, aunque no necesariamente), con todas las instalaciones de una vivienda común (como disponibilidad de cocina y cuarto baño independiente) que ocasionalmente disponen de zonas comunes (como recepción). Los AT no deben confundirse con las denominadas viviendas de uso turístico (VUT), consistentes en viviendas de particulares que son alquiladas con fines turísticos.

Conocer los factores que influyen en el precio de los AT, así como la cuantificación de su valor, puede revelarse interesante para propietarios de este tipo de alojamientos, ayudándoles a fijar una tarifa diaria de la estancia más ajustada a la realidad del mercado. Desde el punto de vista del potencial cliente, puede resultar de utilidad para comprobar si la cuantía por la que se oferta la propiedad es superior o inferior a lo que normalmente se ofrecería en determinadas circunstancias. Asimismo, también podría ser de interés para las múltiples plataformas online a través de las cuales se comercializa este tipo de alojamientos, tales como la anteriormente mencionada Airbnb o Booking.com. Con este objeto, se ha seleccionado el denominado método de precios hedónicos (MPH), dado que, si bien es cierto que existen investigaciones previas sobre aplicación del MPH a la valoración inmobiliaria y establecimientos hoteleros, aún son escasos los estudios que aplican este método a estas 
nuevas modalidades de alojamiento en auge, no siendo encontradas referencias en la literatura métodos de valoración aplicados a los AT.

Por tanto, el objetivo principal de este estudio es conocer los determinantes de valoración en la composición del precio de la estancia por día en los AT, a través de la elaboración de un modelo de precios hedónicos que refleje de manera ajustada qué variables influyen en este tipo de alojamiento y en qué medida. Para ello, se extraen datos de Sevilla, la mayor ciudad del sur de España y una de las ciudades con mayor afluencia turística del país. Para la obtención de la información utilizada en el trabajo se manejaron fuentes tales como el Registro de Turismo Andaluz (RTA), Booking.com y Google Maps. La razón de la selección de Booking.com como fuente principal de datos, fue que ésta dispone de información adicional como la superficie del alojamiento o su ubicación exacta, variables no disponibles en otras plataformas del mismo tipo.

Para la consecución del objetivo propuesto, este estudio se estructura efectuando, en primer lugar, una revisión de los trabajos previos que aplica el MPH en el ámbito de la valoración inmobiliaria y de alojamientos turísticos, para posteriormente desarrollar la metodología en el marco geográfico seleccionado, especificando detalladamente las variables recopiladas. A continuación, se plasman los resultados, discusión y, finalmente, las conclusiones obtenidas al respecto.

\section{REVISIÓN DE LITERATURA}

Dado que este estudio se centra en el análisis del precio de un tipo de inmueble (AT) vinculado al sector turístico, serán examinados los estudios que han aplicado el MPH tanto en la valoración de inmuebles como en el ámbito de los alojamientos turísticos, con especial referencia a aquellos trabajos dedicados a la estimación del precio de las estancias en éstos y las variables que lo determinan.

\subsection{Método de precios hedónicos en valoración inmobiliaria}

Si bien la primera aplicación del MPH al mercado de la vivienda se encuentra en el estudio de Ridker y Henning (1967) sobre la influencia de la polución en el precio de la misma -en el que se destacan variables como los minutos empleados en llegar al centro de la ciudad o CBD (Central Business District)-, es Freeman (1979) el que utiliza la primera justificación teórica del uso del MPH a dicho sector, según las características del vecindario en el que se encuentra el inmueble. Adicionalmente, son reseñables otros trabajos en la misma línea como los de Caridad y Brañas (1996), destacando variables como la superficie del inmueble; Caridad y Ceular (2001), en la que se recoge la disponibilidad de piscina o el barrio en donde se encuentra la vivienda; García Pozo (2008), que incorpora la disponibilidad de garaje como variable relevante; así como Chasco y Le Gallo (2013); Núñez et al. (2013) y Wheeler et al. (2014) en la línea de los anteriores.

Adicionalmente, Nor et al. (2019); Poeta et al. (2019); Bishop et al. (2020) y Kim et al. (2020) resaltan nuevamente características como la superficie y el distrito en donde se ubica el inmueble. Además de ello, se hallan aplicaciones del MPH para locales comerciales, entre los que destacan estudios dedicados exclusivamente a la distancia del inmueble al $C B D$, aeropuerto $u$ otros puntos de interés (Deschermeier et al., 2014); el tipo de uso del local (Özyurt, 2014); o una combinación de ambos tipos de variables (Núñez et al., 2017). Sintetizando, en todos estos estudios inmobiliarios analizados se presentan variables 
esencialmente referidas al tamaño del inmueble, su ubicación dentro de la ciudad, acabados del mismo en términos de calidad, así como la disponibilidad de diferentes atributos adicionales como puede ser la presencia de garaje o trastero incluidos en el precio.

\subsection{Método de precios hedónicos en alojamientos turísticos}

En el terreno de la estimación del precio de las estancias en hoteles utilizando el método de precios hedónicos conviene mencionar, en primer lugar, los de Coenders et al. (2003), Espinet et al. (2003), Rigall et al. (2011) y Saló et al. (2014) -en el que son comparadas estancias hoteleras y segundas viviendas- centrados en el turismo de "sol y playa" desarrollado el litoral mediterráneo español y en Cataluña en especial, resaltando variables relacionadas con la estacionalidad, la disponibilidad de piscina o la distancia a la zona de baño costera. En la misma línea de valoración de tarifas hoteleras, destacan por orden cronológico los trabajos de: Chen y Rothschild (2010) para Taipéi (Taiwán) destacando la superficie de la habitación o la disponibilidad de TV; Andersson (2010) para Singapur incidiendo en el número de estrellas y la disponibilidad de piscina; Zhang et al. (2011) para Nueva York (EE. UU.) resaltando la ubicación del hotel-; Kuminoff et al. (2010) para Virginia (EE. UU.) subrayando la disponibilidad de piscina y número de estrellas; de la Peña et al. (2016) para hoteles en Cuba (en el que es analizada una larga lista de variables entre las que destacan la satisfacción del usuario, disponibilidad de terraza y vistas) y Soler et al. (2019) para Málaga (España) en los que se resaltan variables determinantes como el número de estrellas del hotel, la admisión de mascotas o la valoración de los usuarios. Adicionalmente, otro estudio de Soler (2017) refleja la incidencia en el precio de los hoteles de Sevilla (España) de eventos especiales en la misma como la conocida Feria de Abril.

A diferencia de las fuentes de datos utilizadas en los anteriores -esencialmente TripAdvisor en el caso de Soler et al. (2019) y turoperadores y agencias de viajes para el resto de estudios-, existen algunos trabajos recientes sobre estancias hoteleras que toman a Booking.com como principal recurso. Entre ellos conviene mencionar los siguientes: Castro y Ferreira (2018) en Lisboa (Portugal), centrado en la valoración de usuarios; Latinopoulos (2018) -número de estrellas, parking, piscina- en Halkidiki (Grecia); Mondaca Marino et al. (2019) en Santiago de Chile (Chile); Stojchevska et al. (2018) -balcón, bañera, insonorizaciónen Skopje (Macedonia del Norte); Nieto et al. (2019) en distintas capitales europeas y especializado en diferentes ratings de usuarios en la línea de Castro y Ferreira (2018); Chica Olmo (2020) en Sevilla (distancia a la Giralda y al centro histórico) y Nicolau et al. (2020) para diferentes ciudades a nivel mundial, centrado en variables referidas al número de comentarios, limpieza, o el continente al que pertenece el alojamiento.

En lo que a modelos hedónicos aplicados a viviendas turísticas se refiere, es pionero el estudio de Pérez y Marmolejo (2014) en el destacan variables esencialmente referidas a la disponibilidad de distintas amenidades, o el distrito en el que se encuentra el inmueble. A éste le siguieron otros trabajos que toman a Airbnb como principal fuente de datos como los de Wang y Nicolau (2017) para 33 ciudades europeas y estadounidenses, incluyendo variables como la valoración de los usuarios o distancia al centro de las mismas; Gibbs et al. (2018), desarrollado en diferentes ciudades canadienses, y utilizando la variable número de fotos, o disponibilidad de parking, entre otras; Gunter y Önder (2018) en Viena (Austria) -destacado por la inclusión del número de plazas en el alojamiento como una de sus variables contempladas-; Moreno et al. (2018) en la Comunidad Valenciana (España) y, finalmente Tong y Gunter (2020) en una comparativa de la ciudades españolas de Madrid, Barcelona y Sevilla. 
No se han hallado estudios referidos a apartamentos y viviendas turísticas que utilicen a Booking.com como principal fuente de datos. Con objeto de sintetizar lo anteriormente expuesto, los estudios analizados referidos a establecimientos hoteleros, apartamentos y viviendas turísticas se clasifican según tipos de variables usadas en los mismos en la tabla 1.

Tabla 1. Tipos de variables en los estudios analizados sobre alojamientos turísticos

\begin{tabular}{|c|c|c|}
\hline $\begin{array}{c}\text { Tipo de } \\
\text { variables }\end{array}$ & Descripción & Estudios \\
\hline Tipología & $\begin{array}{l}\text { Categoría del } \\
\text { alojamiento ( } \mathrm{n} \text { - } \\
\text { de estrellas en } \\
\text { hoteles) }\end{array}$ & $\begin{array}{l}\text { Espinet et al. (2003); Andersson (2010); Chen y Rothschild (2010); Kuminoff et } \\
\text { al. (2010); Rigall i Torrent et al. (2011); Saló et al. (2014); De La Peña et al. } \\
\text { (2016); Latinopoulos (2018); Mondaca Marino et al. (2019); Soler et al. (2019); } \\
\text { Chica Olmo (2020) }\end{array}$ \\
\hline Ubicación & $\begin{array}{l}\text { Distancia al } \\
\text { centro u otros } \\
\text { puntos de interés }\end{array}$ & $\begin{array}{c}\text { Espinet et al. (2003); Andersson (2010); Chen y Rothschild (2010); Rigall i } \\
\text { Torrent et al. (2011); Pérez Bastidas y Marmolejo Duarte (2014); Soler García y } \\
\text { Gémar Castillo (2017); Wang y Nicolau (2017); Castro y Ferreira (2018); Gibbs et } \\
\text { al. (2018); Gunter y Önder (2018); Stojchevska et al. (2018); Nieto García et al. } \\
\text { (2019); Soler García et al. (2019), Chica Olmo (2020); Nicolau et al. (2020); Tong } \\
\text { y Gunter (2020) }\end{array}$ \\
\hline Tamaño & $\begin{array}{l}\text { Medido en } \\
\text { superficie o en } \\
\text { número de } \\
\text { ocupantes }\end{array}$ & $\begin{array}{c}\text { Espinet et al. (2003); Chen y Rothschild (2010); Pérez Bastidas y Marmolejo } \\
\text { Duarte (2014); Saló et al. (2014); De La Peña et al. (2016); Wang y Nicolau } \\
\text { (2017); Gibbs et al. (2018); Gunter y Önder (2018); Moreno Izquierdo et al. } \\
\text { (2018); Tong y Gunter (2020) }\end{array}$ \\
\hline Amenidades & $\begin{array}{l}\text { Servicios como } \\
\text { disponibilidad de } \\
\text { TV, parking y } \\
\text { piscina (entre } \\
\text { otras) }\end{array}$ & $\begin{array}{l}\text { Coenders et al. (2003); Espinet et al. (2003); Andersson (2010); Chen y } \\
\text { Rothschild (2010); Kuminoff et al. (2010); Rigall i Torrent et al. (2011); Saló et al. } \\
\text { (2014); Pérez Bastidas y Marmolejo Duarte (2014); Wang y Nicolau (2017); De } \\
\text { La Peña et al. (2016); Castro y Ferreira (2018); Gibbs et al. (2018); Gunter y } \\
\text { Önder (2018); Latinopoulos (2018); Stojchevska et al. (2018); Nieto García et al. } \\
\text { (2019); Soler García et al. (2019); Chica Olmo (2020); Nicolau et al. (2020) }\end{array}$ \\
\hline Experiencia & $\begin{array}{l}\text { Valoración de } \\
\text { usuarios y/o } \\
\text { número de fotos }\end{array}$ & $\begin{array}{l}\text { Wang y Nicolau (2017); Castro y Ferreira (2018); Gibbs et al. (2018); Gunter y } \\
\text { Önder (2018); Mondaca Marino et al. (2019); Moreno Izquierdo et al. (2018); } \\
\text { Stojchevska et al. (2018); Nieto García et al. (2019); Soler García et al. (2019); } \\
\text { Nicolau et al. (2020); Tong y Gunter (2020) }\end{array}$ \\
\hline Estacionalidad & $\begin{array}{l}\text { Temporadas altas } \\
\text { y bajas, eventos } \\
\text { especiales en los } \\
\text { lugares de estudio }\end{array}$ & $\begin{array}{l}\text { Coenders et al. (2003); Espinet et al. (2003); Rigall i Torrent et al. (2011); Saló et } \\
\text { al. (2014); Soler García y Gémar Castillo (2017); Soler et al. (2019) }\end{array}$ \\
\hline
\end{tabular}

Elaboración propia

\section{METODOLOGÍA}

\subsection{Método de precios hedónicos (MPH)}

Según Parker y Zilberman (1993) el método de precios hedónicos (MPH) parte de la base de que el precio de un bien está formado por un agregado del valor de una serie de variables que influyen en éste. De esta forma, conociendo individual y pormenorizadamente esa serie de atributos, así como su ponderación en la determinación final del bien analizado, mediante el uso de técnicas estadísticas de regresión se puede obtener una estimación del precio del bien objeto de análisis asignando valores concretos a las variables que influyen en el mismo.

La mayoría de autores establecen el origen del MPH a partir de los trabajos de Court (1939), debido a que fue el primero en utilizar el término "hedónico" en su estudio acerca de la determinación del aumento de precio de los automóviles. Posteriormente, autores como Griliches (1971) y Rosen (1974) fueron desarrollando un tratamiento unificado del MPH, planteando modelos de oferta y demanda de bienes heterogéneos y sentando las bases del 
enfoque más comúnmente aceptado, popularizándose su uso a partir de entonces en multitud de aplicaciones.

Los modelos econométricos que siguen el MPH se componen de una variable endógena $(Y)$-generalmente, el precio de un bien o servicio-, que se trata de estimar a partir de un número determinado $(k)$ de variables exógenas que influyen causalmente en ésta (Caridad, 1998). Mediante una función $(f)$ que las incluye, se obtiene la estimación de la variable dependiente $(\hat{Y})$, es decir, la parte de $Y$ que puede ser explicada por el conjunto de variables independientes, quedando la diferencia entre ambas reflejada como error o residuo $(e)$.

Las variables exógenas pueden ser de carácter cuantitativo o cualitativo, estas últimas representadas mediante variables artificiales. Adicionalmente, las funciones $(f)$ de los modelos también pueden presentar formas diferenciadas. Siendo las más usuales en el MPH aplicado a alojamientos turísticos la lineal y la semilogarítimica según los modelos previamente observados en la revisión literaria.

\subsection{Marco geográfico y objeto de estudio}

Sevilla es la ciudad más poblada del sur de España (figura 1, A), siendo además la capital de Andalucía, Comunidad Autónoma española en la que se ubica dicha ciudad (Junta de Andalucía, 2019). Su casco antiguo acoge un rico patrimonio cultural con tres monumentos incluidos en la lista Patrimonio de la Humanidad (UNESCO, 2020): La Catedral, el Real Alcázar y el Archivo de Indias, situados alrededor de la Plaza del Triunfo (figura 1, B), que es considerado como el centro turístico. Cuenta, además, con dos eventos de fama mundial que se celebran en abril: la Semana Santa y la Feria.

Figura 1. Ubicación de Sevilla en Europa (A) y alrededores de la Plaza del Triunfo (B)

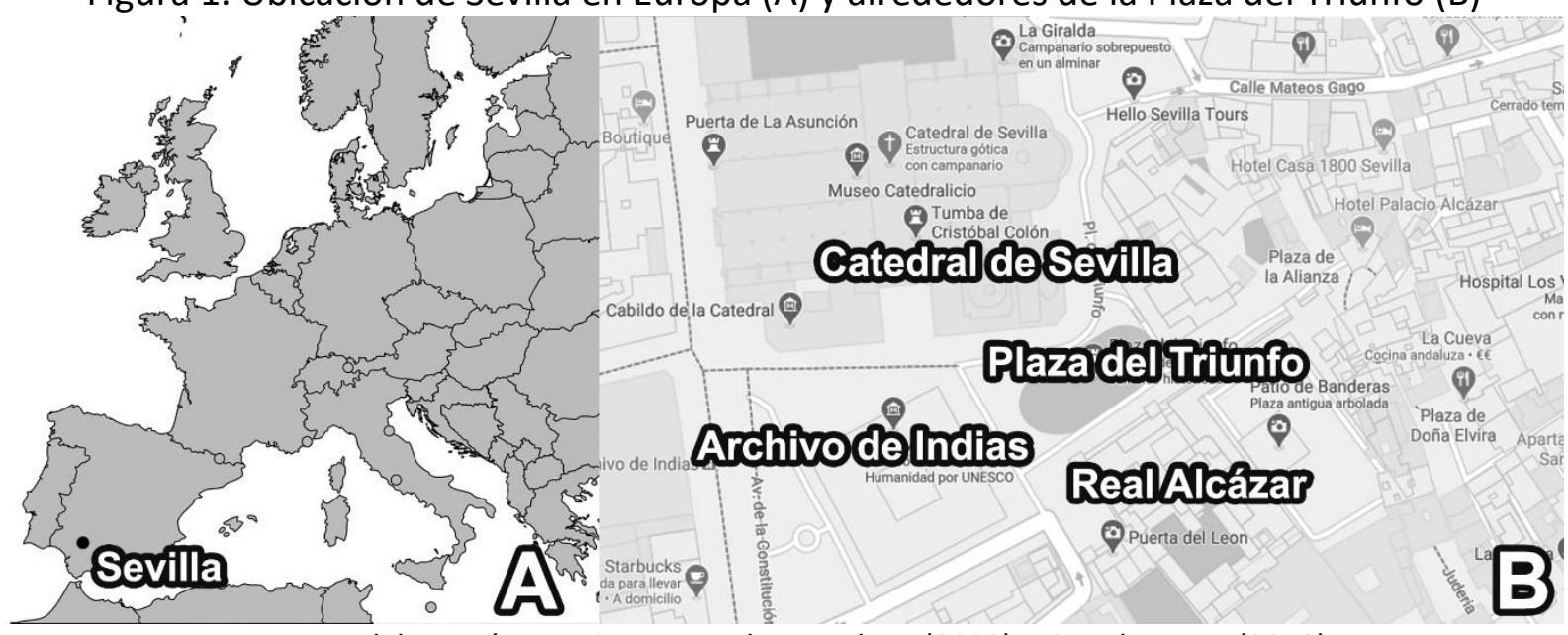

Fuente: elaboración propia a partir de Mapchart (2020) y Google Maps (2018)

Dicha urbe es toda una potencia turística a nivel nacional, siendo la sexta localidad en número de pernoctaciones en España (INE, 2018). Finalmente, conviene resaltar que la prestigiosa guía Lonely Planet (2017), eligió a Sevilla como ciudad número uno para visitar en 2018, a través de su publicación Best in Travel 2018 lanzada en octubre de 2017; y en abril de 2018, la organización internacional del sector turístico WTTC $(2018$, abril 19) anunciaba a Sevilla como sede de la cumbre mundial organizada por esta institución, celebrada los días tres y cuatro de abril de 2019. 
Adicionalmente, la elección de la ciudad de Sevilla como ámbito de análisis del estudio tiene su explicación en el importante incremento de pernoctaciones (nacionales e internacionales) registrado en los diez últimos años en la ciudad, muy por encima de las cifras que corresponden a la región andaluza y a España, tal y como puede apreciarse en la Figura 2. Asimismo, la senda correspondiente a Sevilla, además de mostrar un pronunciado incremento, refleja un carácter estacional menos marcado que el que se manifiesta en los niveles andaluz y español.

Figura 2. Pernoctaciones en AT en términos relativos en Sevilla (2009-2019)

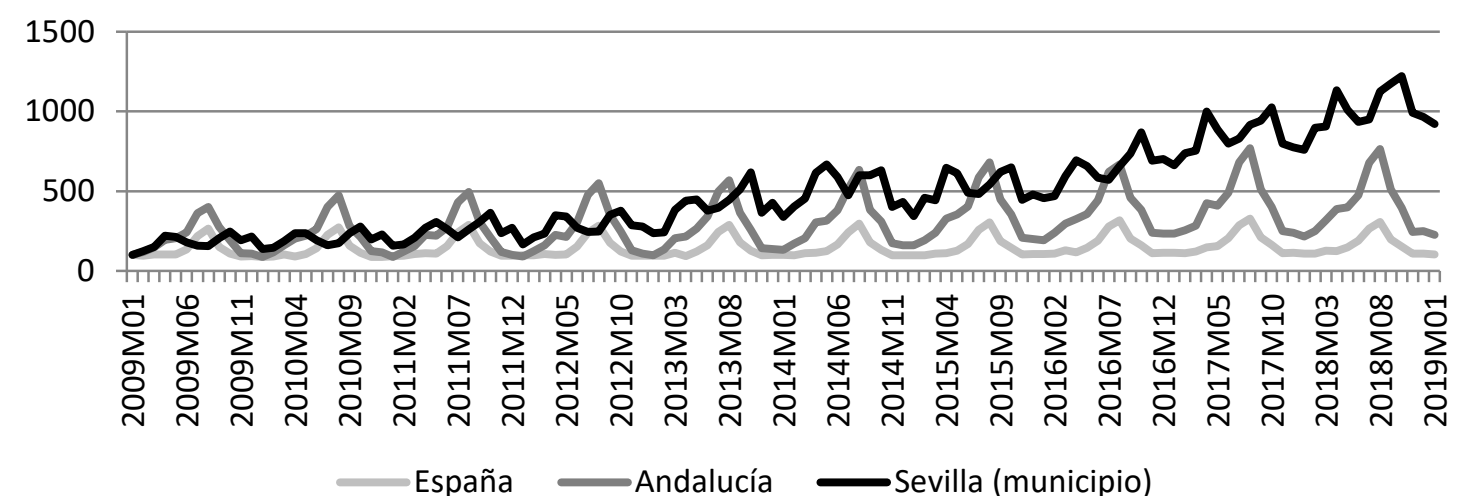

Nota: base enero de $2009=100$. Fuente: elaboración propia a partir de INE (2019) e INE (2019a)

Concretamente para este trabajo se toma como referencia de población total el Registro de Turismo Andaluz (RTA) de todos los AT registrados en el municipio sevillano. La norma que regula los AT en el término municipal de Sevilla es la Ley 13/2011, de 23 de diciembre, del Turismo de Andalucía que los define (art. 44) como "aquellos tipos de alojamientos turísticos compuestos por un conjunto de unidades de alojamiento que cuentan con mobiliario e instalaciones adecuadas para la conservación, elaboración y consumo de alimentos y bebidas; que podrán ser según su tipología constructiva y configuración, apartamentos, villas, chalés, bungalós o inmuebles análogos".

Asimismo, la norma clasifica los AT en dos grupos. Un primer bloque lo conforman los denominados edificio/complejo -que son "aquellos integrados por tres o más unidades de alojamiento que ocupan la totalidad o parte independiente de un edificio o de varios $y$ disponen de entrada propia", mientras que el segundo bloque está formado por los del tipo conjunto, "integrados por tres o más unidades de alojamiento ubicadas en un mismo inmueble o grupo de inmuebles, contiguos o no y que ocupan sólo una parte de los mismos".

Si bien se pretendió que la muestra total analizada coincidiese con la población, este extremo no fue posible, debido a que no se hallaron datos suficientes para alguno de los establecimientos. Así, del total de 133 registros de AT en el municipio de Sevilla (RTA, 2018), sólo pudieron extraerse datos completos de 112 de ellos. Esto originó un total de 610 casos $^{1}$ cuantificándose una muestra total de 2.355 plazas, frente a las 3.734 de la población. La recolección de datos fue realizada durante los meses de septiembre, octubre y noviembre de 2018.

\footnotetext{
${ }^{1}$ Debido a que de cada registro se tomaron tantos apartamentos cómo fue posible, y de éstos, se incluyeron datos del mismo apartamento ofertado para distinto número de ocupantes. 


\subsection{Especificaciones de las variables seleccionadas}

A partir de la revisión de literatura anteriormente efectuada, se obtiene una referencia acerca de las variables que más influyen en el precio de los alojamientos turísticos evidenciadas en estudios previos, tales como aquellos determinantes referidos a la tipología de inmueble, ubicación del mismo, tamaño, amenidades que ofrece, así como aquellos aspectos relacionados con la experiencia y la estacionalidad (ver Tabla 1). La Tabla 2 resume todas las variables consideradas en este estudio y sus estadísticos descriptivos. Se utiliza la web de reservas Booking.com (2018) como fuente principal de datos, ya que ofrece información acerca de la superficie o de la ubicación exacta de la propiedad, aspectos a priori de sumo interés para la estimación de precios y que no se reflejan en otros portales como Airbnb.

Tabla 2. Variables consideradas y sus estadísticos descriptivos

\begin{tabular}{|c|c|c|c|c|c|c|c|}
\hline Variable & Tipo & Descripción & Media o \% & $\begin{array}{l}\text { Desv. } \\
\text { típica }\end{array}$ & Min. & Máx. & Fuente \\
\hline PRE & V. dependiente & $\begin{array}{l}\text { Precio diario en } \\
\text { euros (obtenido } \\
\text { en búsquedas } \\
\text { para dos días de } \\
\text { estancia) }\end{array}$ & 159,81 & 79,47 & 47,00 & 477,85 & $\begin{array}{c}\text { Booking.com } \\
\text { (2018) }\end{array}$ \\
\hline$G R P$ & \multirow{2}{*}{ Tipología } & $\begin{array}{c}\text { Grupo } \\
\text { (edificio/complej } \\
\text { o, conjunto) }\end{array}$ & $\begin{array}{c}\text { Edificio/complejo } \\
(58,03 \%), \text { conjunto } \\
(41,97 \%)\end{array}$ & - & - & - & RTA (2018) \\
\hline$C A T$ & & $\begin{array}{c}\text { Categoría ( } \mathrm{n} \text { o de } \\
\text { Llaves) }\end{array}$ & $\begin{array}{c}1 \text { Llave }(34,3 \%), \\
2 \text { Llaves }(62,10 \%), \\
3 \text { Llaves }(3,6 \%)\end{array}$ & - & - & - & RTA (2018) \\
\hline DIS & \multirow{2}{*}{ Ubicación } & Distrito & $\begin{array}{c}\text { Casco Antiguo } \\
(97 \%), \text { Triana } \\
(1,5 \%), \text { Otros } \\
(1,5 \%) \\
\end{array}$ & - & - & - & $\begin{array}{c}\text { Booking.com } \\
\text { (2018) }\end{array}$ \\
\hline MIN & & $\begin{array}{c}\text { Minutos } \\
\text { caminando hasta } \\
\text { la Plaza del } \\
\text { Triunfo }\end{array}$ & 11,47 & 9,67 & 2 & 94 & $\begin{array}{c}\text { Google Maps } \\
\text { (2018) }\end{array}$ \\
\hline M2 & \multirow[b]{2}{*}{ Tamaño } & Superficie $\left(\mathrm{m}^{2}\right)$ & 63,06 & 40,28 & 15 & 600 & \multirow{15}{*}{$\begin{array}{c}\text { Booking.com } \\
\text { (2018) }\end{array}$} \\
\hline OCU & & $\begin{array}{l}\text { Número de } \\
\text { ocupantes }\end{array}$ & 3,86 & 1,82 & 1 & 16 & \\
\hline$T V$ & \multirow{11}{*}{ Amenidades } & Televisión & $99,30 \%$ & - & - & - & \\
\hline LAV & & Lavadora & $75,60 \%$ & - & - & - & \\
\hline$B A L$ & & Balcón & $33,80 \%$ & - & - & - & \\
\hline TER & & Terraza & $32,60 \%$ & - & - & - & \\
\hline PAT & & Patio & $18,90 \%$ & - & - & - & \\
\hline VIS & & Vistas & $46,40 \%$ & - & - & - & \\
\hline INS & & Insonorización & $30,20 \%$ & - & - & - & \\
\hline$P A R$ & & Aparcamiento & $63,10 \%$ & - & - & - & \\
\hline MAS & & $\begin{array}{c}\text { Admisión de } \\
\text { mascotas }\end{array}$ & $12,80 \%$ & - & - & - & \\
\hline PIS & & Piscina & $9,30 \%$ & - & - & - & \\
\hline$B A \tilde{N}$ & & Bañera & $14,10 \%$ & - & - & - & \\
\hline$V A L$ & \multirow{2}{*}{ Experiencia } & $\begin{array}{c}\text { Valoración de } \\
\text { usuarios }\end{array}$ & 8,78 & 0,58 & 6,50 & 9,80 & \\
\hline FOT & & Número de fotos & 43,33 & 6,95 & 15 & 47 & \\
\hline
\end{tabular}




\begin{tabular}{|c|c|c|c|c|c|c|c|}
\hline$A V I$ & & Atractivo visual & 8,71 & 0,82 & 5,00 & 10,00 & $\begin{array}{c}\text { Elaboración } \\
\text { propia }\end{array}$ \\
\hline TAES & \multirow{6}{*}{ Estacionalidad } & $\begin{array}{c}\text { Temporada alta, } \\
\text { entre semana } \\
(27 / 05 / 19- \\
29 / 05 / 19) \\
\end{array}$ & $31,64 \%$ & - & - & - & \multirow{6}{*}{$\begin{array}{l}\text { Booking.com } \\
\text { (2018) }\end{array}$} \\
\hline TAFS & & $\begin{array}{c}\text { Temporada alta, } \\
\text { fin de semana } \\
(31 / 05 / 19- \\
02 / 06 / 19)\end{array}$ & $14,75 \%$ & - & - & - & \\
\hline TBES & & $\begin{array}{c}\text { Temporada baja, } \\
\text { entre semana } \\
(14 / 01 / 19- \\
16 / 01 / 19)\end{array}$ & $29,02 \%$ & - & - & - & \\
\hline TBFS & & $\begin{array}{c}\text { Temporada baja, } \\
\text { fin de semana } \\
(18 / 01 / 19- \\
20 / 01 / 19)\end{array}$ & $12,95 \%$ & - & - & - & \\
\hline SS & & $\begin{array}{c}\text { Semana Santa } \\
(18 / 04 / 19- \\
20 / 04 / 19) \\
\end{array}$ & $6,56 \%$ & - & - & - & \\
\hline$F E$ & & $\begin{array}{c}\text { Feria } \\
(10 / 05 / 19- \\
12 / 05 / 19)\end{array}$ & $5,08 \%$ & - & - & - & \\
\hline
\end{tabular}

A continuación, se detallan los criterios utilizados para la extracción de los datos en algunas de las variables consideradas. En primer lugar, la variable precio (PRE) recoge la cuantía reflejada en euros en Booking.com (2018) por alojamiento y día, contabilizado para una estancia de dos días, ya que es ésta la media para la ciudad de Sevilla según el Centro de Datos Turísticos del Ayuntamiento de Sevilla (2017). En el caso de que un edificio/complejo o conjunto de AT oferte distintas unidades de alojamiento al mismo precio, se escoge siempre aquella opción que presenta un mayor valor añadido en lo que a sus características se refiere, siguiendo el comportamiento de un consumidor racional.

La categoría (CAT) del AT es medida en número de llaves (de una a cuatro) de forma similar al número de estrellas en los hoteles. Para el ámbito analizado, no se encontraron establecimientos de cuatro llaves. Asimismo, se añaden también los minutos (MIN) que se emplean en recorrer caminando la distancia comprendida entre el alojamiento y la Plaza del Triunfo (ver Figura 1, B). La elección de este lugar es debida a su consideración como punto de interés máximo para el visitante, por estar ubicada entre los dos monumentos más visitados de la ciudad según el Centro de Datos Turísticos del Ayuntamiento de Sevilla (2017), es decir, entre la Catedral de Sevilla y el Real Alcázar (figura 1, B).

Tras el visionado de las fotos de la propiedad, sólo son consideradas las vistas (VIS) a la ciudad y/o monumentos emblemáticos, nunca a patios y/o jardines interiores. Con respecto a la disponibilidad de aparcamiento $(P A R)$ se contabilizan tanto los de propiedad del AT como los próximos a éste, aunque no fueran de su titularidad. Adicionalmente, se añade una valoración propia por parte de los autores acerca del atractivo visual (AVI) del alojamiento, en escala de cero a diez, basada en criterios objetivos tales como el orden, la limpieza y/o la calidad mostrada en las fotografías.

Finalmente, el precio se recoge en distintos momentos del tiempo. Se han considerado seis intervalos de dos días (tabla 2). Para su ponderación, se tuvo en cuenta una división 
aproximada en dos mitades iguales en cuanto a las temporadas alta y baja (ya que cada una ocupa unos seis meses del año $^{2}$ ) otorgando un peso mayor a la temporada alta, debido al consecuente aumento de las pernoctaciones en la misma. Respecto a los fines de semana, suponen algo más de las dos séptimas partes de los casos si se comparan con la toma entre semana, como consecuencia del lógico aumento de las pernoctas durante esos días. Por último, para los eventos especiales de Semana Santa y Feria, de una semana de duración aproximadamente cada uno de ellos, se toma la referencia de triplicar y duplicar respectivamente el $2 \%$ que supone una semana sobre el total del año, ya que se produce un aumento significativo de pernoctas en estos periodos del año.

\section{RESULTADOS}

A partir de la muestra obtenida con 610 casos se elabora un modelo mediante el método de precios hedónicos (MPH). Como se mencionó anteriormente, para su especificación pueden utilizarse diversas formas funcionales. No existe un criterio determinante que permita seleccionar a priori cuál es la mejor forma para la función hedónica. Tras efectuar diferentes pruebas con otras formas funcionales, se selecciona la función lineal por ser la que arrojaba mejores resultados atendiendo al coeficiente de determinación obtenido y a los distintos contrastes de validación. Los programas utilizados han sido SPSS de IBM Corp. y EViews.

Adicionalmente, a través del método de las distancias de Cook, se observan doce casos en los que, analizadas sus características a nivel individual, se consideran originados por edificios singulares, distintos a la mayoría de los apartamentos en oferta, y, por tanto, se eliminan de la muestra. Se aplica además el principio de parsimonia, según el cual, entre diferentes modelos con grado de ajuste similar siempre se elige el obtenido con un número menor de variables explicativas, seleccionando finalmente las que se presentan en la Ecuación 1.

$$
\begin{gathered}
P R E=-86,04+17,16 G R P-1,95 M I N+0,61 M 2+10,09 O C U+12,94 T E R+ \\
16,79 I N S+27,04 P I S+12,16 V A L-1,20 F O T+11,80 A V I+23,54 T A F S-
\end{gathered}
$$

$25,20 T B E S-18,88 T B F S+168,78 S S+112,68 F E+e$

La primera observación que ha de hacerse es que, en el caso de la estacionalidad, se toma como base la variable temporada alta entre semana (TAES) excluyéndose por tanto del modelo, ya que su inclusión presentaría un problema de multicolinealidad exacta en el mismo. Seguidamente, los coeficientes (ecuación 1) informan acerca de las variaciones marginales que se producen en el precio (variable dependiente) ante modificaciones de cada una de las variables independientes. Así, como puede apreciarse, las máximas variaciones en el precio tienen un origen estacional, concretamente durante Semana Santa y Feria de Abril se detectan ascensos en las tarifas diarias de $168,78 €$ y $112,68 €$, respectivamente, en base al precio tomado en temporada alta entre semana (TAES).

Por otro lado, se han detectado variables que tienen una relación decreciente con el precio de la tarifa, como el decremento de $25,20 €$ que se produce en el precio en temporada baja entre semana (TBES) con respecto al de temporada alta entre semana. Además de ello, existen otras variables con influencia en el precio: cada minuto extra empleado en caminar desde el alojamiento a la Plaza del Triunfo reduce en 1,95€ el precio diario del mismo, mientras que cada metro cuadrado del que disponga lo aumenta en $0,61 €$. Añadiendo otros

\footnotetext{
${ }^{2}$ Considerando temporada alta la que comprende desde abril a septiembre, y baja de octubre a marzo.
} 
ejemplos, un AT con terraza disponible incrementa su tarifa por día en $12,94 €$ con respecto a uno sin la misma, análogamente, ocurre lo mismo con respecto a la disponibilidad de piscina en $27,04 €$.

De esta forma, el precio diario de la estancia en AT bajo unas condiciones determinadas es fácilmente estimable. Conocidas previamente todas las variables seleccionadas como relevantes en la ecuación 1, su aplicación en la misma nos dará lugar a una tarifa determinada a estos factores fijados con antelación. Esta función es especialmente interesante para conocer si un precio ofertado se encuentra infravalorado o sobrevalorado con respecto a lo que ofrecería el mercado bajo esas circunstancias.

La tabla 3 presenta un análisis pormenorizado de las variables seleccionadas y de sus coeficientes. En ésta, se muestran adicionalmente los errores estándar obtenidos, así como los estadísticos $T$ y su probabilidad límite $p$, que en ningún caso superó el 0,01 , lo que refleja que todas las variables incorporadas al modelo son estadísticamente significativas. Asimismo, se realizaron pruebas adicionales que descartaron problemas de multicolinealidad entre variables explicativas; el Factor de Inflación de la Varianza (FIV, tabla 3) no rebasó el nivel 10 para ninguna variable exógena.

Tabla 3. Variables del modelo lineal mediante MPH

\begin{tabular}{|c|c|c|c|c|c|}
\hline Variable & Coeficiente & $\begin{array}{c}\text { Error } \\
\text { estándar }\end{array}$ & $T$ & $p$ & $F I V$ \\
\hline Constante & $-86,035$ & 32,702 & $-2,631$ & 0,009 & - \\
\hline GRP $=$ Edificio/complejo & 17,163 & 3,820 & 4,493 & 0,000 & 1,158 \\
\hline MIN (minutos caminando hasta la Plaza del Triunfo) & $-1,950$ & 0,324 & $-6,019$ & 0,000 & 1,137 \\
\hline M2 (superficie) & 0,607 & 0,075 & 8,070 & 0,000 & 2,131 \\
\hline OCU (Número de ocupantes) & 10,087 & 1,378 & 7,319 & 0,000 & 2,043 \\
\hline TER (Terraza) & 12,942 & 3,809 & 3,398 & 0,001 & 1,050 \\
\hline INS (Insonorización) & 16,790 & 4,030 & 4,166 & 0,000 & 1,127 \\
\hline PIS (Piscina) & 27,036 & 6,497 & 4,161 & 0,000 & 1,111 \\
\hline VAL (Valoración de usuarios) & 12,164 & 3,977 & 3,059 & 0,002 & 1,627 \\
\hline FOT (Níumero de fotos) & $-1,198$ & 0,271 & $-4,425$ & 0,000 & 1,086 \\
\hline AVI (Atractivo visual) & 11,801 & 2,870 & 4,112 & 0,000 & 1,673 \\
\hline TAFS (Temporada alta, fin de semana) & 23,543 & 5,607 & 4,199 & 0,000 & 1,297 \\
\hline TBES (Temporada baja, entre semana) & $-25,195$ & 4,693 & $-5,369$ & 0,000 & 1,470 \\
\hline TBFS (Temporada baja, fin de semana) & $-18,875$ & 5,892 & $-3,203$ & 0,001 & 1,283 \\
\hline SS (Semana Santa) & 168,772 & 7,519 & 22,446 & 0,000 & 1,150 \\
\hline FE (Feria) & 112,676 & 8,609 & 13,088 & 0,000 & 1,114 \\
\hline
\end{tabular}

Con respecto al grado del ajuste del modelo obtenido, se obtiene un coeficiente de determinación $\left(R^{2}\right)$ de 0,713 . Las medidas de ajuste de Akaike y de Schwartz son las menores para el modelo propuesto, respecto a varios alternativos. El error relativo medio (ERM) obtenido -la diferencia media absoluta de los valores reales frente a los estimados en términos relativos-, asciende a un 20,37\%. Por último, el índice de desigualdad de Theil fue estimado en 0,1198 . Su proximidad a cero revela una elevada capacidad predictiva del modelo. 
De manera gráfica, la Figura 3 refleja una comparativa entre los valores de los precios reales frente a los estimados por el modelo. De existir un ajuste perfecto, la nube de puntos se posicionaría en torno a la bisectriz del primer cuadrante. La forma presentada por la nube de puntos se sitúa en torno a dicha línea, lo que es indicativo de un buen grado de ajuste. No obstante, se observa que el modelo tiende a infravalorar para tarifas diarias superiores a $300 €$ aproximadamente.

Figura 3. Precio real vs. precio estimado por el modelo

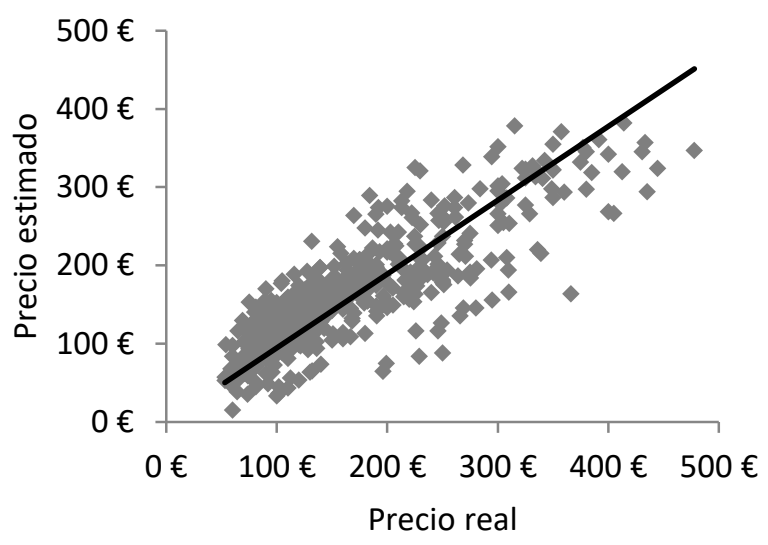

\section{DISCUSIÓN}

En primer lugar, con respecto a la variable (MIN), cada minuto extra empleado en caminar desde el alojamiento hasta el centro turístico (Plaza del Triunfo) rebaja el precio del mismo. Es decir, como cabía esperar, existe una relación inversa entre la distancia al centro histórico de la ciudad y el precio de la pernoctación. A diferencia de otras investigaciones que toman la distancia al centro como una variable cualitativa (Espinet et al., 2003), o medida en distancia (Soler y Gémar, 2017) este estudio mide en tiempo empleado caminando, de forma que sea más fácilmente interpretable por los consumidores y otros agentes económicos.

El modelo especificado recoge los dos eventos más reseñables para la ciudad como Semana Santa (SS) y Feria de Abril (FE), los cuales suponen respectivamente unos incrementos en el precio diario de la estancia de $168,78 €$ y $112,68 €$. Estas dos variables se posicionan como las más influyentes en el precio, jugando un papel primordial en la construcción del modelo, en la línea del patrón propuesto por Soler y Gémar (2017).

Adicionalmente, la disponibilidad de piscina (PIS), en un ámbito de estudio caracterizado por unas condiciones climáticas extremas durante el verano, también se revela como un factor destacado. Estudios anteriores (Rigall et al., 2011) también demostraron la influencia de la disponibilidad de piscina en el precio. Además de ello, la insonorización (INS) también se muestra como relevante, muy apreciada en grandes ciudades turísticamente muy concurridas. El grupo (GRP) de AT destaca como significativo, reflejando una preferencia por la tipología edificio/complejo, que habitualmente dispone de zonas comunes (como recepción) frente al tipo conjunto, en el que las unidades de alojamiento pueden encontrarse en diferentes bloques, dificultando la disponibilidad de las mencionadas zonas comunes.

Secundariamente, aparecen variables referidas al atractivo visual (AVI) y las valoraciones de anteriores usuarios (VAL), sendas con influencia similar en el precio según lo revelado por el modelo. Con respecto al número de fotos (FOT) resulta paradójico observar 
que este factor que a priori podría considerarse como una variable que influiría positivamente en el precio -tal y como sucede en Gibbs et al. (2018)- (ya que un número mayor de fotos parecería indicar un interés del propietario en mostrar el atractivo visual del alojamiento) resulta, sin embargo, negativo. Una posible explicación de esta situación puede ser que un número elevado de imágenes incrementa la posibilidad de que alguna de ellas recoja un aspecto que disguste o desagrade al cliente potencial y, por ende, el precio que esté dispuesto a desembolsar disminuya. Otra explicación podría encontrarse en la saturación del potencial usuario ante una cantidad excesiva de fotos. Adicionalmente, factores como la superficie (M2), poco utilizada en la literatura revisada referente a alojamientos turísticos -en la que generalmente suele emplearse el número de habitaciones u ocupantes-, y la ubicación (MIN), aparecen como determinantes en la composición del precio de AT.

\section{CONCLUSIONES}

El notable incremento del turismo en la última década a nivel mundial en general, así como en determinadas regiones en particular, ha provocado la aparición de nuevos tipos de alojamientos turísticos. Concretamente este trabajo se ha focalizado en la valoración de la modalidad de apartamento turístico, sin encontrarse estudios previos de valoración de este tipo de alojamiento. Dicha modalidad, ha experimentado un reciente auge espectacular en España, por lo que se ha seleccionado Sevilla para el desarrollo del presente estudio, una ciudad con elevada afluencia turística, y en la que este tipo de alojamiento presenta una fuerte incidencia desde los últimos años.

La revisión de literatura realizada permite sustentar las hipótesis subyacentes al modelo propuesto sobre la base principal del Método de Precios Hedónicos (MPH), en el que el precio de la estancia es estimado a partir de una suma ponderada de un conjunto de atributos relacionados principalmente con la tipología-categoría del alojamiento, su ubicación, tamaño -expresado tanto en superficie como en número de ocupantes, aspecto especialmente valorado en reservas para familiares u otros grupos-, amenidades (como la terraza, apreciada en zonas con climatología cálida), experiencia del usuario o factores relacionados con la estacionalidad (temporada alta y baja), el día de la semana de la estancia (entre semana, fin de semana), así como los eventos especiales en la ciudad (Semana Santa y Feria de Abril). Por lo que respecta a la ubicación, y a diferencia de la literatura abordada, se consideró desde un primer momento imprescindible incluir en el modelo una referencia medida en tiempo y no en distancia, lo que facilita al consumidor una interpretación en términos de coste más clara de la variable.

Con respecto a la valoración del modelo MPH en su conjunto, puede concluirse la obtención de un coeficiente de determinación $\left(R^{2}=0,713\right)$ y un $\operatorname{ERM}(20,37 \%)$ que permite obtener unos resultados con un óptimo ajuste de estimación de precios, con una forma funcional en la línea de la literatura abordada (Kuminoff et al., 2010; Wang y Nicolau, 2017; Soler et al., 2019); así como con una cantidad no excesiva de variables suficientemente relevantes y diferentes entre sí, que adicionalmente no presentan multicolinealidad apreciable entre ellas.

El modelo desarrollado es especialmente útil para conocer si una tarifa diaria de estancia en un AT con unos determinados atributos y bajo unas condiciones determinadas se encuentra en la línea de lo que el mercado ofrecería bajo estas circunstancias específicas o no. Esta función puede resultar de interés a los principales agentes del sector tales como 
potenciales turistas, propietarios del alojamiento, o plataformas online, que actúan como intermediarias.

Las principales limitaciones al presente estudio fueron las referentes a la falta de disponibilidad de datos en algunos casos o a la existencia de información incompleta en otros de ellos. Finalmente, como futuras líneas de investigación pueden señalarse la inclusión de nuevas variables que mejoren el grado de ajuste del modelo o bien adaptar la metodología usada en este estudio a otras ciudades lo que permitirá efectuar estudios comparativos acerca de las variables determinantes en la valoración.

\section{REFERENCIAS BIBLIOGRÁFICAS}

Andersson, D.E. (2010). Hotel attributes and hedonic prices: an analysis of internet-based transactions in Singapore's market for hotel rooms. The Annals of Regional Science, 44(2), 229-240.

Bishop, K.C., Kuminoff, N.V., Banzhaf, H.S., Boyle, K.J., Von Gravenitz, K., Pope, J.C., Smith, V.K. y Timmins, C.D. (2020). Best practices for using hedonic property value models to measure willingness to pay for environmental quality. Review of Environmental Economics and Policy, 14(2), 260-281.

Booking.com. (2018). Sevilla. Apartamentos. Recuperado de https://www.booking.com/searchresults.es.html?aid=304142\&label=gen173nr1FCAEoggJCAlhYSDNYBGhGiAEBmAEKwgEKd2luZG93cyAxMMgBDNgBAegBAfgBC5ICA XmoAgM\&sid=ea1464c3588d729c4d34d0887be0ddc6\&class_interval=1\&dest_id=402849\&dest_type=city\&from_sf=1\&group_adults=2 .

Caridad y Ocerin, J.M. (1998). Econometría: modelos econométricos y series temporales. Tomo I. Barcelona: Reverté.

Caridad y Ocerin, J.M. y Brañas Garza, P. (1996). Demanda de características de la vivienda en Córdoba: Un modelo de precios hedónico. Revista de Estudios Regionales(46), 139153.

Caridad y Ocerin, J.M. y Ceular Villamandos, N. (2001). Un análisis del mercado de la vivienda a través de redes neuronales artificiales. Estudios de Economía Aplicada (18), 67-81.

Castro, C. y Ferreira, F. A. (2018). Online hotel ratings and its influence on hotel room rates: the case of Lisbon, Portugal. Tourism \& Management Studies, 14, 63-72.

Centro de Datos Turísticos del Ayuntamiento de Sevilla. (Eds.). (2017). Informe Anual 2017. Sevilla: Centro de Datos Turísticos del Ayuntamiento de Sevilla.

Chasco, C. y Le Gallo, J. (2013). The Impact of Objective and Subjective Measures of Air Quality and Noise on House Prices: A Multilevel Approach for Downtown Madrid. Economic Geography, 89(2), 127-148.

Chen, C-F. y Rothschild, R. (2010). An application of hedonic pricing analysis to the case of hotel rooms in Taipei. Tourism Economics, 16(3), 685-694.

Chica Olmo, J. (2020). Effect of monumental heritage sites on hotel room pricing. International Journal of Hospitality Management, 90, 102640. 
Coenders, G., Espinet, J. y Saez, M. (2003). Predicting random level and seasonality of hotel prices: a latent growth curve approach. Tourism Analysis, 8(1), 15-31.

Court, A.T. (1939). Hedonic price indexes with automotive examples. En General Motors Corporation (Eds.), The Dynamics of Automobile Demand (pp. 99-117). Detroit: General Motors Corporation.

de la Peña, M.R., Núñez-Serrano, J.A., Turrion, J. y Velázquez, F.J. (2016). Are innovations relevant for consumers in the hospitality industry? A hedonic approach for Cuban hotels. Tourism Management, 55, 184-196.

Deschermeier, P., Voigtländer, M. y Seipelt, B. (2014). Modelling a hedonic index for commercial properties En ERES (Eds.), European Real Estate Society 21st Annual Conference (pp. 1-12). Bucarest: European Real Estate Society (ERES).

Espinet, J.M., Saez, M., Coenders, G. y Fluvià, M. (2003). Effect on prices of the attributes of holiday hotels: a hedonic prices approach. Tourism Economics, 9(2), 165-177.

Freeman, A.M. (1979). The hedonic price approach to measuring demand for neighborhood characteristics. En S. D. Segal (Eds.), The Economics of Neighborhood: Studies in Urban Economics (pp. 191-217). Nueva York: Academic Press.

García Pozo, A. (2008). Determinantes del precio de la vivienda usada en Málaga: Una aplicación de la metodología hedónica. Revista de Estudios Regionales (82), 135-158.

Gibbs, C., Guttentag, D., Gretzel, U., Morton, J. y Goodwill, A. (2018). Pricing in the sharing economy: a hedonic pricing model applied to Airbnb listings. Journal of Travel \& Tourism Marketing, 35(1), 46-56.

Google Maps. (2018). Sevilla. Recuperado de https://www.google.es/maps/place/Sevilla/@37.3846082,5.9944208,15z/data $=! 4 \mathrm{~m} 5 ! 3 \mathrm{~m} 4 ! 1 \mathrm{~s} 0 \times \mathrm{xd} 126 \mathrm{c} 1114 \mathrm{be} 6291: 0 \times 34 \mathrm{f0} 18621 \mathrm{cfe} 5648 ! 8 \mathrm{~m} 2 ! 3$ d37.3890924! $4 \mathrm{~d}-5.9844589$ ?hl=es

Griliches, Z. (1971). Introduction: Hedonic Price Indexes Revisited. In Griliches, Z. (Eds.) Price Indexes and Quality Changes: Studies in New Methods of Measurement. Cambridge: Harvard University Press.

Gunter, U. y Önder, I. (2018). Determinants of Airbnb demand in Vienna and their implications for the traditional accommodation industry. Tourism Economics, 24(3), 270-293.

INE. (2018). Indicadores Urbanos. Recuperado de https://www.ine.es/prensa/ua_2018.pdf _

INE. (2019). Encuesta de ocupación en apartamentos turísticos. Viajeros y pernoctaciones por $\begin{array}{lll}\text { puntos turísticos } & \text { (Sevilla). Recuperado de }\end{array}$ https://www.ine.es/jaxiT3/Datos.htm?t=2082

INE. (2019a). Encuesta de ocupación en apartamentos turísticos. Viajeros y pernoctaciones por comunidades autónomas. Recuperado de https://www.ine.es/jaxiT3/Datos.htm?t=1993

Junta de Andalucía. (2019). Instituto de Estadística y Cartografía de Andalucía IECA. Recuperado de http://www.juntadeandalucia.es/institutodeestadisticaycartografia/sima/ficha.htm? mun=41091 
Kim, G.S., Schieffer, J. y Mark, T. (2020). Do superfund sites affect local property values? Evidence from a spatial hedonic approach. Economic Analysis and Policy, 67, 15-28.

Kuminoff, N.V., Zhang, C. y Rudi, J. (2010). Are travelers willing to pay a premium to stay at a "green" hotel? Evidence from an internal meta-analysis of hedonic price premia. Agricultural and Resource Economics Review, 39(3), 468-484.

Latinopoulos, D. (2018). Using a spatial hedonic analysis to evaluate the effect of sea view on hotel prices. Tourism Management, 65, 87-99.

Ley 13/2011, de 23 de diciembre, del Turismo de Andalucía. Boletín Oficial de la Junta de Andalucía, no 255, de 31 de diciembre de 2011, pp. 3 a 22.

Lonely Planet. (Eds.) (2017). Lonely Planet's Best in Travel 2018. Melbourne: Lonely Planet.

Mapchart. (2020). Europe. Recuperado de https://mapchart.net/europe.html

Mondaca Marino, C., Guala, C., Montecinos Astorga, A.L. y Salazar Concha, C. (2019). Factores que Influyen en el Precio de Hoteles en Booking.com. El Caso de Santiago de Chile. Información Tecnológica, 30(1), 87-96

Moreno Izquierdo, L., Egorova, G., Peretó Rovira, A. y Más Ferrando, A. (2018). Exploring the use of artificial intelligence in price maximisation in the tourism sector: its application in the case of Airbnb in the Valencian Community. Investigaciones Regionales, 42, 113128.

Nicolau, J.L., Mellinas, J.P. y Martín-Fuentes, E. (2020). Satisfaction measures with monetary and non-monetary components: hotel's overall scores. International Journal of Hospitality Management, 87, 102497.

Nieto García, M., Resce, G., Ishizaka, A., Occhiocupo, N. y Viglia, G. (2019). The dimensions of hotel customer ratings that boost RevPAR. International Journal of Hospitality Management, 77, 583-592.

Nor, M.I., Masron, T.A. y Gedi, S.Y. (2019). Modeling of residential property rents in Somalia using two-stage modeling. International Journal of Housing Markets and Analysis, 13(2), 331-356.

Núñez Tabales, J.M., Rey Carmona, F.J. y Caridad y Ocerin, J.M. (2013). Precios implícitos en valoración inmobiliaria urbana. Revista de la construcción, 12(2), 116-126.

Núñez Tabales, J.M., Rey Carmona, F.J. y Caridad y Ocerin, J.M. (2017). Redes neuronales (RN) aplicadas a la valoración de locales comerciales. Informes de la Construcción (IDLC), 69(545), 1-10. e179.

Özyurt, S. (Eds.) (2014). Spatial dependence in commercial property prices: micro evidence from the Netherlands. Frankfurt: European Central Bank.

Parker, D. y Zilberman, D. (1993). Hedonic estimation of quality factors affecting the farmretail margin. American Journal of Agricultural Economics, 75(2), 458-466.

Pérez Bastidas, V.B. y Marmolejo Duarte, C.R. (2014). El impacto de las externalidades producidas por el turismo sobre los valores inmobiliarios y la segmentación del mercado residencial en Barcelona. ACE: Architecture, City and Environment, 9(25), 159188. 
Poeta, S., Gerhardt, T. y Stumpf Gonzalez, M. (2019). Análisis de precios hedónicos de viviendas. Revista ingeniería de construcción, 34(2), 215-220.

Ridker, R.G. y Henning, J.A. (1967). The Determinants of Residential Property Values with Special Reference to Air Pollution. The Review of Economics and Statistics, 49(2), 246257.

Rigall i Torrent, R., Fluvià, M., Ballester, R., Saló, A., Ariza, E., y Espinet, J.M. (2011). The effects of beach characteristics and location with respect to hotel prices. Tourism Management, 32(5), 1150-1158.

Rosen, S. (1974). Hedonic prices and implicit markets: product differentiation in pure competition. Journal of political economy, 82(1), 34-55.

RTA. (Eds.). (2018). Registro de Turismo de Andalucía. AT, VFT y VTAR. Sevilla: Consejería de Turismo y Deporte.

Saló, A., Garriga, A., Rigall-i-Torrent, R., Vila, M. y Fluvià, M. (2014). Do implicit prices for hotels and second homes show differences in tourists' valuation for public attributes for each type of accommodation facility? International Journal of Hospitality Management, 36, 120-129.

Soler García, I.P. y Gémar Castillo, G. (2017). Impact of the April Fair on Seville hotel room prices: measurement through a hedonic approach. Tourism \& Management Studies, 13(2), 7-12.

Soler García, I.P. y Gémar Castillo, G., Correia, M.B. y Serra, F. (2019). Algarve hotel price determinants: A hedonic pricing model. Tourism Management, 70, 311-321.

Stojchevska, M., Naumoski, A., y Mitreski, K.. (2018). Modelling the Impact of the Hotel Facilities on Online Hotel Review Score for City of Skopje. En IEEE (Eds.). 2nd International Symposium on Multidisciplinary Studies and Innovative Technologies (ISMSIT) (pp. 1-5). Ankara: IEEE.

Tong, B. y Gunter, U. (2020). Hedonic pricing and the sharing economy: how profile characteristics affect Airbnb accommodation prices in Barcelona, Madrid, and Seville. Current Issues in Tourism, 1-20

UNESCO. (2020). United Nations Educational, Scientific and Cultural Organization. Recuperado de https://whc.unesco.org/en/statesparties/es

UNWTO. (2019). International Tourism Highlights. 2019 Edition. Recuperado de https://www.e-unwto.org/doi/pdf/10.18111/9789284421152

Wang, D., y Nicolau, J.L. (2017). Price determinants of sharing economy based accommodation rental:A study of listings from 33 cities on Airbnb.com. International Journal of Hospitality Management, 62, 120-131.

Wheeler, D.C., Páez, A., Spinney, J. y Waller, L.A. (2014). A Bayesian approach to hedonic price analysis. Papers in Regional Science, 93(3), 663-683.

WTTC. (2018, abril 19). 2019 WTTC Global Summit host announced: SEVILLE, SPAIN. Recuperado de https://www.wttc.org/about/media-centre/press-releases/pressreleases/2018/2019-wttc-global-summit-host-announced-seville-spain/ 
Zhang, Z., Ye, Q. y Law, R. (2011). Determinants of hotel room price: An exploration of travelers' hierarchy of accommodation needs. International Journal of Contemporary Hospitality Management, 23(7), 972-981.

\section{CONTRIBUCIONES DE LOS AUTORES:}

Autor 1: Concepción original del trabajo, metodología, configuración y ajuste de la base de datos, análisis estadístico y validación, interpretación de los resultados, redacción y revisión crítica del contenido y aprobación de la versión a publicar.

Autor 2: Concepción original del trabajo, metodología, interpretación de los resultados, redacción y revisión crítica del contenido y aprobación de la versión a publicar.

Autor 3: Metodología, configuración y ajuste de la base de datos, análisis estadístico y validación, redacción y revisión crítica del contenido y aprobación de la versión a publicar. 